\title{
GA-ANFIS PID compensated model reference adaptive control for BLDC motor
}

\author{
Murali Dasari ${ }^{1}$, A Srinivasula Reddy ${ }^{2}$, M Vijaya Kumar ${ }^{3}$ \\ ${ }^{1,3}$ Department of Electrical and Electronics Engineering, JNTU College of Engineering, India \\ ${ }^{2} \mathrm{CMR}$ Engineering College, Hyderabad, Telangana, India
}

\begin{tabular}{l}
\hline \hline Article Info \\
\hline Article history: \\
Received Jun 17, 2018 \\
Revised Nov 5, 2018 \\
Accepted Dec 10, 2018 \\
\hline Keywords: \\
ANFIS \\
BLDC \\
GA \\
MRAC \\
PID \\
\hline
\end{tabular}

\begin{abstract}
Adaptive control is one of the widely used control strategies to design advanced control systems for better performance and accuracy. Model reference adaptive control (MRAC) is a direct adaptive strategy with some adjustable controller parameters and an adjusting mechanism to adjust them. In this work Model Reference Adaptive Control for BLDC motors has been designed with a PID controller tuned by GA-ANFIS. GA-Trained ANFIS framework for tuning the PID controller has been proposed. This is used along with the MRAC to deliver enhanced performance in the control of BLDC motor. The performance of the proposed approach is validated for motor control under conditions of change in speed, change in load, change in inertia and change in phase resistance. The performance is validated against convention PID and self tuning PID controllers. The result demonstrates a superior performance of the proposed approach
\end{abstract}

Copyright (c) 2019 Institute of Advanced Engineering and Science. All rights reserved.

\section{Corresponding Author:}

Murali Dasari,

Department of Electrical and Electronics Engineering,

JNTU College of Engineering, Ananthpur, Andhra, India.

Email: Pradesh,muralidvr@gmail.com

\section{INTRODUCTION}

Control of BLDC motor has always remained an active area of research. Especially the PID control of BLDC motor has been studied extensively for the optimization of different parameters of proportional gain, integral time and derivative time. Researchers have used different optimization approaches to optimize these parameters. Researchers have drawn inspiration from naturally occurring phenomena in solving these optimization problems. mimicking the behavior of natural systems (or) naturally occurring phenomena have given rise to multiple optimization approaches like Particle Swarm Optimization (PSO) [1] Ant Colony Optimization (ACO) [2] Genetic Algorithm (GA) [3] Bacterial Foraging Optimization Algorithm (BFOA) Differential evolution (DE) [4] Immune Algorithm (IA) [5] etc. These algorithms have adapted from naturally occurring process. They can be referred using different names with the names like Evolutionary Algorithms and metaheuristic approaches being commonly used. The metaheuristic approaches typically combine heuristic algorithms which are usually problem specific in a more generalized frame work. So, metaheuristics can be considered as processes which strategies to find an optimum (or) a near optimum solution. These metaheuristic approaches are approximate and non-deterministic and they usually employ mechanisms to have a good convergence and provide near optimum solutions.

Similarly, ANFIS is a very effective modeling approach which combines the attributes of both the fuzzy inference system and neural network. The amalgamation of fuzzy logic with architectural design of neural network led to creation of neuro- fuzzy systems. A multitude of methods have been used to optimize the fuzzy membership functions in the literature. These methods can be divided into two types including derivative based and heuristic algorithms in general [6]. Shoorehdeli et al [7], [8] proposed hybrid methods composed particle swarm optimization (PSO). He used recursive least square (RLS) and extended Kalman 
filter (EKL) for training. In different studies, they proposed factor recursive least square for training the conclusion parameters and Lyapunov stability theory to improve the performance of ANFIS. In addition to these, they used NSGA-II the training of all parameters of ANFIS structure. Similarly Zangeneh et al [9] proposed a new type of training ANFIS [10], [11] is applying complex type (DE/current-tobest/ 1+1/bin \& $\mathrm{DE} / \mathrm{rand} / 1 / \mathrm{bin}$ ) on predicting of Mackeyglass time series.

In this paper we have proposed GA-Trained ANFIS framework for tuning the PID controller. This controller is used in unison with a Model Reference Adaptive Control (MRAC) to achieve control of a BLDC motor. The performance of the proposed controller is evaluated against a standard self tuning PID [12]-[13] controller for tracking the speed changes due to sudden change in load, sudden change in reference speed., sudden change in inertia and phase resistance. The detailed model of BLDC [14] is explained in section 2. Section 3 explains about GA-ANFIS system, here the performance of a PID controller is compared for tuning through ANFIS and GA ANFIS. The GA ANFIS MARC setup is explained in section 4, while section 5 briefs about the results.

\section{MODELING OF BLDC MOTOR}

The mathematical model of BLDC has lot of similarities to conventional DC motors. One major addition is in regard to the phases which impact the overall functioning and efficiency of the BLDC. These phases typically exert their influence on resistive and inductive arrangement of BLDC. The schematic BLDC is illustrated in Figure 1.

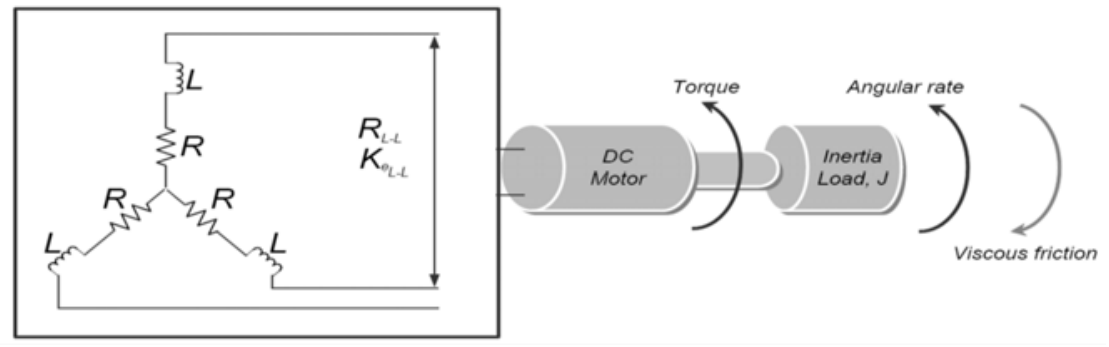

Figure 1. Schematic of BLDC

The mathematical model of DC motor is

$$
\mathrm{G}(\boldsymbol{s})=\frac{\frac{1}{K_{e}}}{\tau_{m} \cdot \tau_{e} \cdot \boldsymbol{s}^{2}+\tau_{m} \cdot s+1}
$$

The mechanical time constant is

$$
\tau_{m}=\frac{R J}{K_{e} K_{t}}
$$

The electrical time constant

$$
\tau_{e}=\frac{L}{R}
$$

In the case of the BLDC motors the constants take these forms (4) and (5)

$$
\begin{aligned}
& \tau_{m}=\frac{R \cdot J}{K_{e} \cdot K_{t}}=\frac{J \sum R}{K_{e} \cdot K_{t}} \\
& \tau_{e}=\sum \frac{L}{R}=\frac{L}{\sum R}
\end{aligned}
$$

Since there is a symmetrical arrangement and a three phase, the mechanical (known) and electrical constants become (6) and (7):

Int J Pow Elec \& Dri Syst, Vol. 10, No. 1, March 2019 : 265 - 276 


$$
\begin{aligned}
& \tau_{m}=\frac{J .3 R}{K_{e} \cdot K_{t}} \\
& \tau_{e}=\frac{L}{3 . R}
\end{aligned}
$$

Considering the phase effects,

$$
\begin{aligned}
\tau_{m} & =\frac{3 \cdot R_{\emptyset} J}{\left(\frac{K_{e(L-L)}}{\sqrt{3}}\right) \cdot K_{t}} \\
\tau_{m} & =\frac{3 \cdot R_{\varnothing} J}{K_{e} \cdot K_{t}}
\end{aligned}
$$

where $K e$ is the phase value of the EMF (voltage) constant;

$$
K_{e}=\frac{K_{e(L-L)}}{\sqrt{3}}
$$

Also, there is a relationship between $K_{e}$ and $K_{t}$; using the electrical power (left hand side) and mechanical power (right hand side) equations;

$$
\begin{aligned}
& \sqrt{3} \times E \times I=\frac{2 \pi}{60} \times N \times T \\
& \frac{E}{N}=\frac{T}{I} \times \frac{2 \pi \times 1}{60 \times \sqrt{3}} \\
& K_{e}=K_{t} \times \frac{2 \pi \times 1}{60 \times \sqrt{3}} \\
& K_{e}=K_{t} \times 0.0605
\end{aligned}
$$

where $\boldsymbol{K}_{\boldsymbol{e}}=\left[\frac{v-\boldsymbol{s e c s}}{\operatorname{rad}}\right]$, the electrical torque and $\boldsymbol{K}_{\boldsymbol{t}}=\left[\frac{N-\boldsymbol{m}}{\boldsymbol{A}}\right]$ is the mechanical torque. Considering the effects of the constants and the phase, the equation for BLDC can be obtained as,

$$
G(s)=\frac{\frac{1}{K_{e}}}{\tau_{m} \cdot \tau_{e} \cdot s^{2}+\tau_{m} \cdot s+1}
$$

\section{GA ANFIS PID CONTROLLER}

Fuzzy logic as an idea proposed by Zadeh was first implemented by Madani in the year 1975 [14]. Madani demonstrated the idea of implementing the fuzzy logic as a concept for use in model steam engine. Subsequently many applications evolved using the concept of fuzzy logic. Different applications of fuzzy logic for industrial and home applications can be found in the literature. Two important factors namely, selection of knowledge techniques and availability of knowledge base influence the design of Fuzzy Logic Controllers. These two factors primarily influence the applications of Fuzzy logic. This can be overcome with the use of Adaptive Neuro -Fuzzy Inference System (ANFIS). An adaptive Neuro -Fuzzy Inference System (ANFIS) is a combination of an Artificial Neural Network (ANN) and a fuzzy inference system (FIS).ANN emulates the functioning of human brain and is formulated as collection of artificial neurons. An adaptive network has multiple layers of feed forward network. In this topology each node of the multilayer network executes specific functions on incoming signals. Each node has its own specific function. In the case of adaptive network two types of nodes namely adaptive and fixed nodes are present. In the case of Sugeno FIS the output membership functions are singleton spikes.

In the case of Genetic Algorithm, the initial chromosomes which are randomly populated are referred to as parent chromosomes and subsequent generations of chromosomes are referred to as child (or) offspring. The principle behind genetic algorithm is to involve better parents in the process of reproduction, so as to improve the chances of producing better offspring. Through this process of natural selection, the stronger chromosomes are carried forward to the next stage while the weaker chromosomes are eliminated.

ANFIS delivers an efficient performance in system identification and delivers good prediction and control performance too. The performance of the ANFIS system can be improved through training and GA enhanced training is one of the most preferred and suitable method. GA enhanced training is more suitable 
because in the case of ANFIS the training has to imparted to both the antecedent part and conclusion part of the parameters. The Gaussian membership function is as depicted in (16)

$$
\mu_{A_{I}}(X)=\frac{1}{1+\left[\left(\frac{X-C_{i}}{a_{i}}\right)^{2}\right] b_{i}}
$$

Where $\left\{a_{i}, b_{i}, c_{i}\right\}$ are the parameters of MFS which are affected in shape of MFs. $a_{i}$ is the variance of the membership function, $c_{i}$ the center of membership function and $b_{i}$ is usually equal to 1 .In the antecedent part there are 3 set of trainable parameters which has $\mathrm{N}$ genes. $\mathrm{N}$ is the number of Membership Functions. The optimization algorithm also trains the conclusion part which has $(I+1) \times R$ genes, where $R$ denotes the number of rules and I denotes the number of dimensions of data inputs. The fitness is defined as Root Mean Square Error (RMSE). Parameters are initialized randomly in first step and then are being updated using GA algorithm. In each iteration, one of the parameters set are being updated. i.e. in first iteration for example $a_{i}$ are updated then in second iteration $b_{i}$ are updated and then after updating all parameters again the first parameter update is considered.

In order to test the performance of the proposed approach a test Simulink system is constructed. The system has a PID controller which is tuned by the ANFIS and GA-ANFIS approaches. The Simulink model of the proposed GA-Fuzzy controller is given in Figure 2.

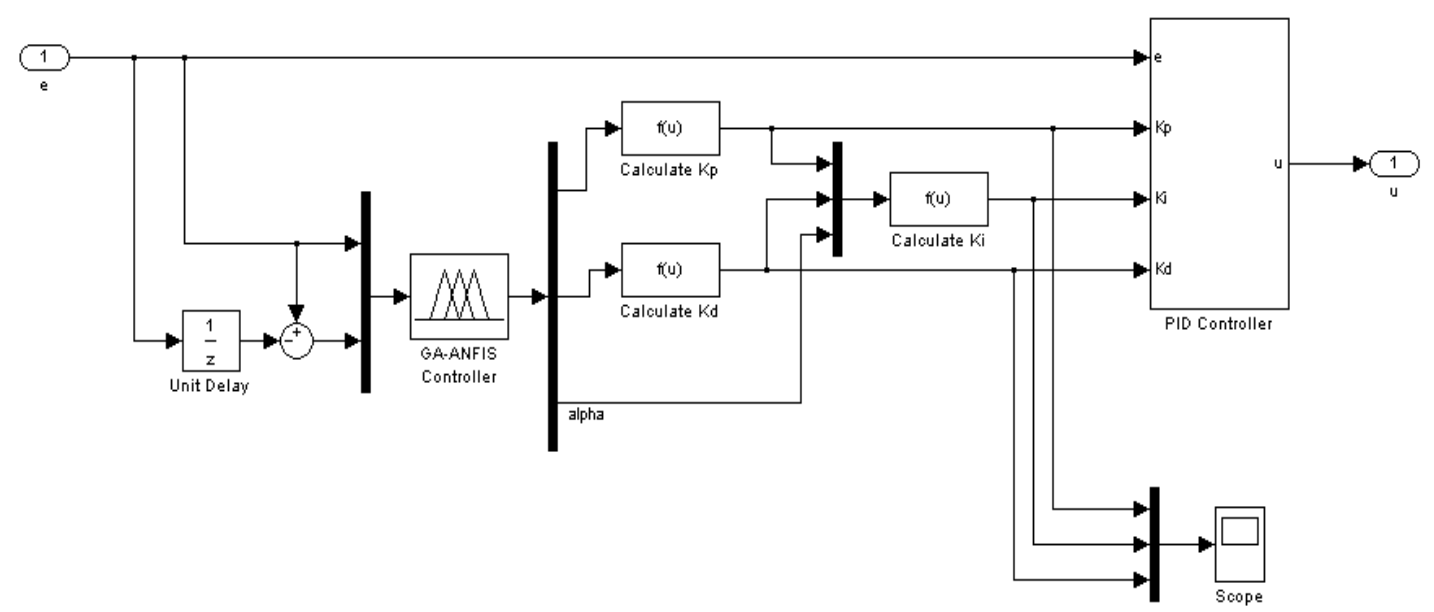

Figure 2. Simulink model of the GA-ANFIS controller setup

The distribution of mean error for the both the methods are illustrated with the help of Figure 3 (a) \& (b). During training ANFIS produced a mean error of -3.8201 with a standard deviation of 25.34 while GA-ANFIS produced a mean error of 4.6434 and a standard deviation of 23.0026.

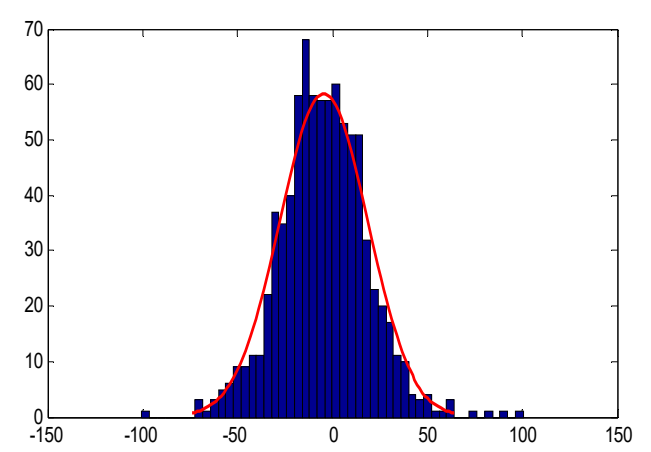

(a)

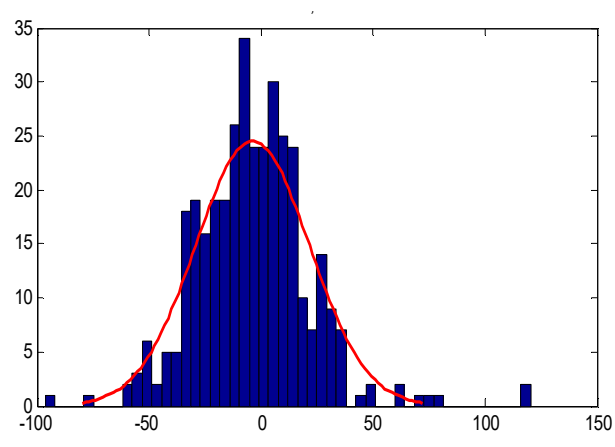

(b)

Figure (3) (a) Distribution of mean error - ANFIS, (b) Distribution of mean error-GA-ANFIS 
It can be clearly observed from the Figure 4, that the settling time and the peak overshoot of the GAANFIS tuned PID is much lesser when compared to the settling time and the overshoot experienced by the ANFIS tuned PID controller for the proposed BLDC motor.

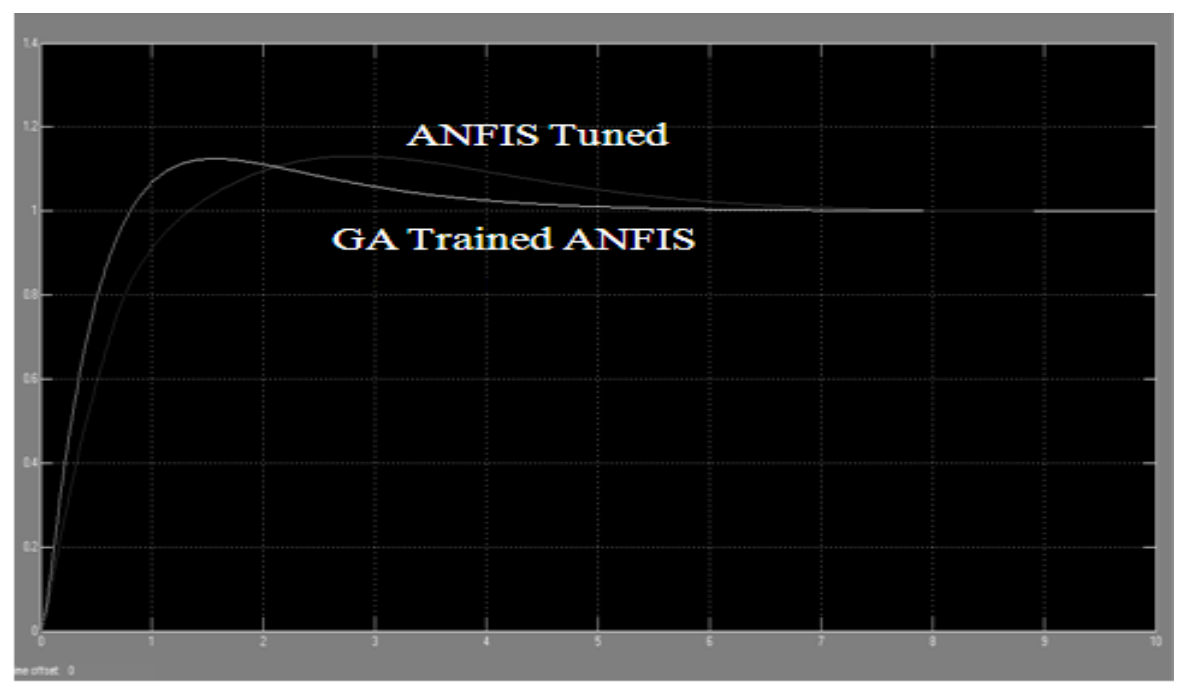

Figure 4. Controller response for ANFIS PID controller and GA-ANFIS PID controller of BLDC motor

\section{MARC GA-ANFIS PID CONTROLLER}

Model Reference Adaptive Control (MRAC) is a direct adaptive strategy with some adjustable controller parameters and an adjusting mechanism to adjust them. As compared to the well-known and simple structured fixed gain PID controllers, adaptive controllers are very effective to handle the unknown parameter variations and environmental changes. An adaptive controller consists of two loops, an outer loop or normal feedback loop and an inner loop or parameter adjustment loop as indicate in Figure 5. Figure 6 shows block diagram of the proposed system. Model Reference Adaptive Control strategy is used to design the adaptive controller that works on the principle of adjusting the controller parameters so that the output of the actual plant tracks the output of a reference model having the same reference input. The MIT rule has been employed here and rule a cost function is defined as;

$$
J(\theta)=\frac{1}{2} e^{2}
$$

To make $\mathbf{J}$ small, it is reasonable to change the parameters in the direction of the negative gradient of $\mathrm{J}$, that is,

$$
\frac{d \theta}{d t}=-\gamma \frac{\partial J}{\partial \theta}=-\gamma e \frac{\partial e}{\partial \theta}
$$

where; $\gamma$ : Adaptation gain., $\theta$ : The controller parameter.

e: The error between the output speed of the BLDC motor and the model reference output. in Figure 7.

It assumed that the process is described by the single-input, single-output (SISO) system as shown 


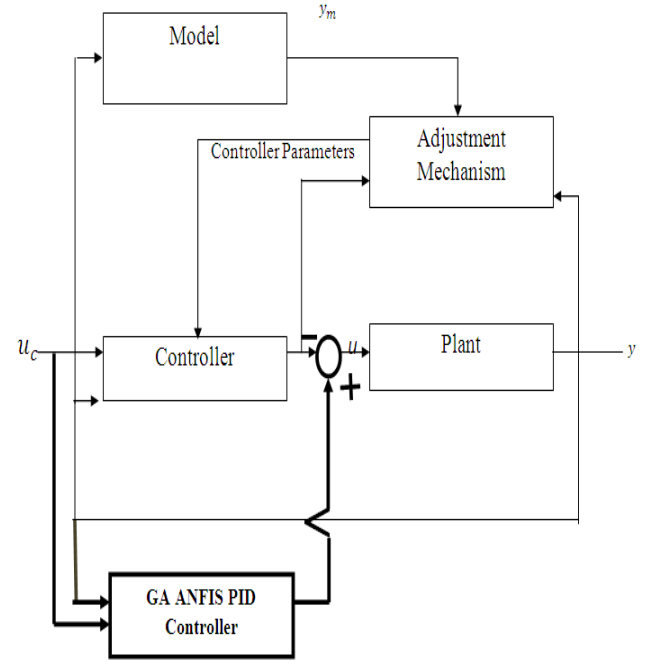

Figure 5. The proposed MARC controller setup

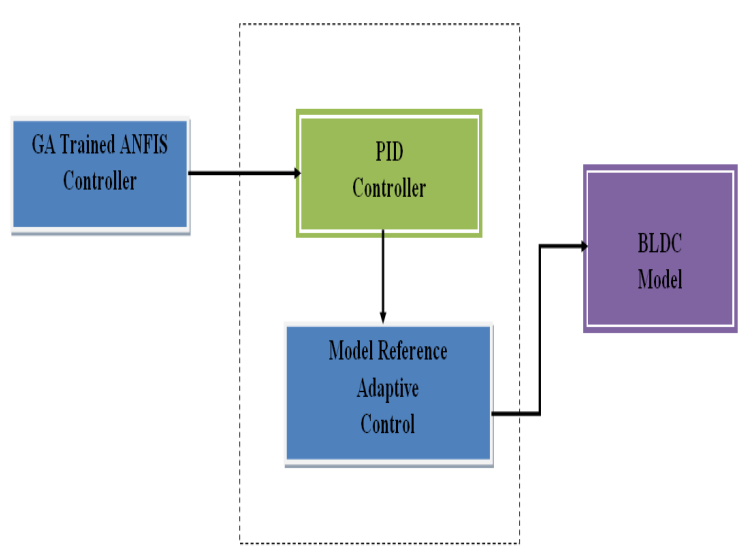

Figure 6. Block diagram of the proposed system

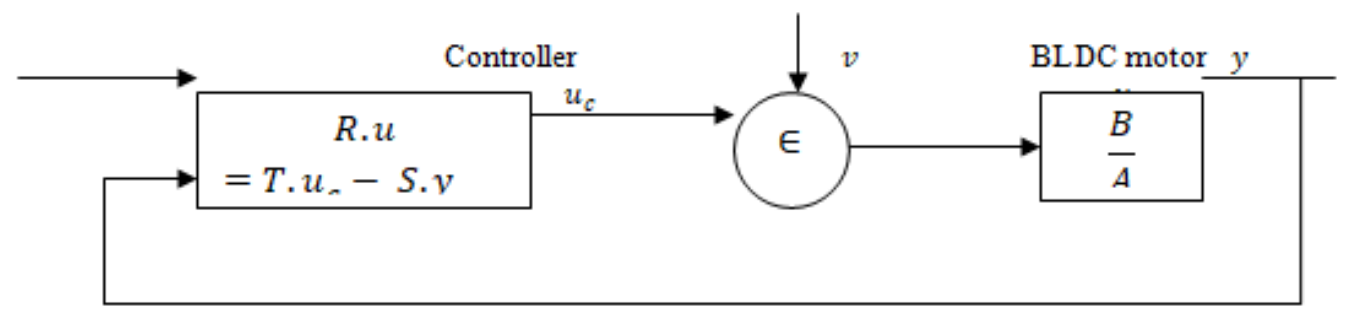

Figure 7. A general linear controller with two degrees of freedom

$$
A \cdot y(t)=B(u(t)+v(t))
$$

where: $A \& B$ are polynomials depend on the BLDC motor. , $(\mathrm{t})$ : The output of controller.

$y(t)$ : The output speed of BLDC motor. $v(t)$ : The process disturbance.

The controller is described in (20)

$$
R u(t)=T u_{c}(t)-S y(t)
$$

where: $R, T$ and $S$ are controller polynomials. , $\boldsymbol{u}_{\boldsymbol{c}}(\boldsymbol{t})$ : The desired speed of BLDC motor.

Substituting (11) into (10) will result (12)

$$
y(t)=\frac{B T}{A R+B S} u_{c}(t)+\frac{B R}{A R+B S} v(t)
$$

Assume the model reference is described by the single-input, single-output (SISO) system

$$
A_{m}(t) y_{m}(t)=B_{m} u_{c}(t)=>y_{m}(t)=\frac{B_{m}}{A_{m}} u_{c}(t)
$$

where: $\boldsymbol{A}_{\boldsymbol{m}}$ and $\boldsymbol{B}_{\boldsymbol{m}}$ are polynomials depend on the reference model. , $\boldsymbol{y}_{\boldsymbol{m}}(\boldsymbol{t})$ : The output of model reference. Assuming, $(\mathrm{V}(t)=0)$ the following condition must hold:

$$
\begin{aligned}
& y(t)=y_{m}(t)=>\frac{B T}{A R+B S}=\frac{B_{m}}{A_{m}} \\
& \frac{y_{m}}{u_{c}}=\frac{b_{m}}{a_{m 1} P^{2}+a_{m 2} P+a_{m 3}}
\end{aligned}
$$


where: $P=\frac{d}{d t}, \boldsymbol{a}_{m 1}, \boldsymbol{a}_{m 2}, \boldsymbol{a}_{m 3}, \boldsymbol{b}_{m}$ : The model reference transfer function coefficient.

Assume the transfer function of the BLDC motor is

$$
\frac{y}{u}=\frac{b}{a_{1} P^{2}+a_{2} P+a_{3}}
$$

Where; $a_{1}, a_{2}, a_{3}$ BLDC motor transfer function coefficient. The Diophantine equation is

$$
A R+B S=\boldsymbol{A}_{\mathbf{0}} \boldsymbol{A}_{\boldsymbol{m}}
$$

Where: $A=a_{1} P^{2}+a_{2} P+a_{3}, A_{m}=a_{m 1} P^{2}+a_{m 2} P+a_{m 3}$

And $\boldsymbol{A}_{\mathbf{0}}$ is a gain., $\mathrm{R}$ and $\mathrm{S}$ : controller polynomials.

$$
\operatorname{deg}(S)=\operatorname{deg}(A)-1=2-1=1
$$

Where deg is the polynomial degree.

$$
\begin{aligned}
& S=s_{0}+P s_{1} \\
& \operatorname{deg}(R)=\operatorname{deg}(S)=>R=r_{0}+r_{1} \mathrm{P} \\
& \operatorname{deg}\left(A_{0}\right)=\operatorname{deg}(A)+\operatorname{deg}(R)-\operatorname{deg}\left(A_{m}\right)=2+1-2=1 \\
& A_{0}=P
\end{aligned}
$$

Similarly $\mathrm{T}=\mathrm{P}$

Substituting (27) to (21) will result (31)

$\left(r_{0}+r_{1} \mathrm{P}\right) \mathrm{u}=\mathrm{P} u_{c}-\left(s_{1} P+s_{0}\right) y$

$u=\frac{P}{R(P)} u_{c}-\frac{S(P)}{R(P)} y$

From (10) and assume $v(t)=0$

$\left(a_{1} P^{2}+a_{2} P+a_{3}\right)=b u$

Substituting (32) into (33) will result (34)

$$
\begin{aligned}
& \left(a_{1} P^{2}+a_{2} P+a_{3}\right) \mathrm{y}=\mathrm{b}\left(\frac{T(P)}{R(P)} u_{c}-\frac{S(P)}{R(P)} \mathrm{y}\right) \\
& =>\left(\left(a_{1} P^{2}+a_{2} P+a_{3}\right)+\mathrm{b} \frac{S(P)}{R(P)}\right) y=b \frac{T(P)}{R(P)} u_{c}
\end{aligned}
$$

Modifying (34) to become (35)

$$
\begin{aligned}
& y=\frac{b T(P)}{\left(a_{1} P^{2}+a_{2} P+a_{3}\right) R(P)+b S(P)} u_{c} \\
& e=y-y_{m}
\end{aligned}
$$

Substituting (24), (35) into (36) will result (37)

$$
\begin{aligned}
& e=\left(\frac{b T(P)}{\left(a_{1} P^{2}+a_{2} P+a_{3}\right) R(P)+b S(P)}-\frac{b_{m}}{a_{m 1} P^{2}+a_{m 2} P+a_{m 3}}\right) u_{c} \\
& \frac{\partial e}{\partial T}=\frac{b}{\left(a_{1} P^{2}+a_{2} P+a_{3}\right) R(P)+b S(P)} u_{c}
\end{aligned}
$$




$$
\frac{\partial e}{\partial S}=\frac{-b^{2} T(P)}{\left(\left(a_{1} P^{2}+a_{2} P+a_{3}\right) R(P)+b S(P)\right)^{2}} u_{c}
$$

From (15)

$$
\begin{aligned}
& \frac{\partial T}{\partial t}=-\gamma e \frac{b}{\left(a_{1} P^{2}+a_{2} P+a_{3}\right) R(P)+b S(P)} u_{c} \\
& \frac{\partial T}{\partial t}=-\gamma^{\prime} e \frac{1}{\left(a_{1} P^{2}+a_{2} P+a_{3}\right) R(P)+b S(P)} u_{c}
\end{aligned}
$$

where $\gamma^{\prime}=b \gamma$

Similarly

$$
\begin{aligned}
\frac{\partial T}{\partial t} & =-\gamma^{\prime} e \frac{1}{a_{m 1} P^{2}+a_{m 2} P+a_{m 3}} \mathrm{y} \\
\frac{B_{m}}{A_{m}} & =\frac{\omega_{n}^{2}}{P^{2}+2 \xi \omega_{n P+\omega_{n}^{2}}}
\end{aligned}
$$

where: $\xi($ damping ratio $)=1 . \omega_{n}($ natural frequency $)=500 .($ selected by designer $)$

\section{RESULTS AND DISCUSSION}

In order to validate the performance of the proposed controller setup is subjected to different test cases, like sudden change in load and sudden change in speed. The results of which are presented here.

The Figure 8 illustrates the open loop response of the designed BLDC model. In order to validate the model a sudden load change is applied at 0.1 seconds. Initially the motor is run at no load and the speed curve points to the no load operation. Then at $\mathrm{t}=0.1$ second a load equivalent to the $50 \%$ of the rated load is applied. It can be observed that there is a sudden drop in speed at that instant and the motor settles at lesser speed from $\mathrm{t}=0.14$ seconds.

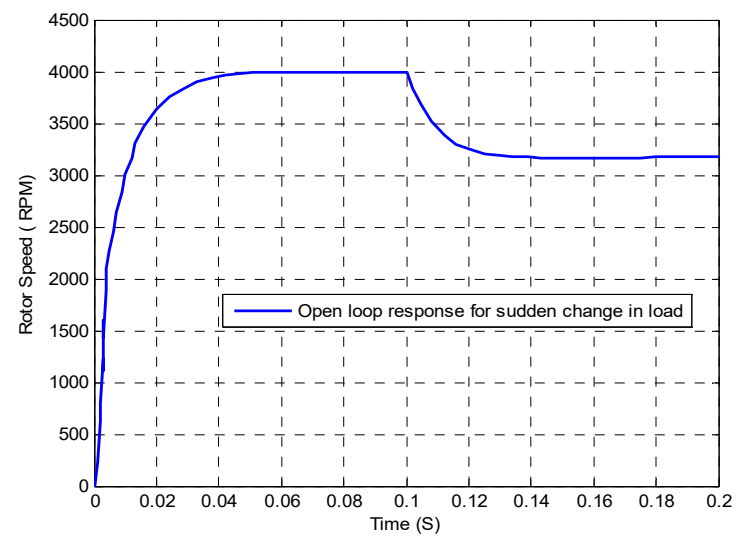

Figure 8. Plot of open loop speed response at sudden change in load

The Figure 9 illustrates the speed response of the controller for different adaptation rate gain, the speed regulation characteristics are studied with the sudden change in load. The load torque is varied to $50 \%$ of the rated value at $\mathrm{t}=0.25$ second. This is after initial settling time for the controller. This graph demonstrates the suitability of higher adaptation rate on the performance of the controller. It can be observed from the figure that higher adaptation gain reduces the rise time. It can also be inferred that it also reduces the overshoot and steady state error.

Int J Pow Elec \& Dri Syst, Vol. 10, No. 1, March 2019 : 265 - 276 


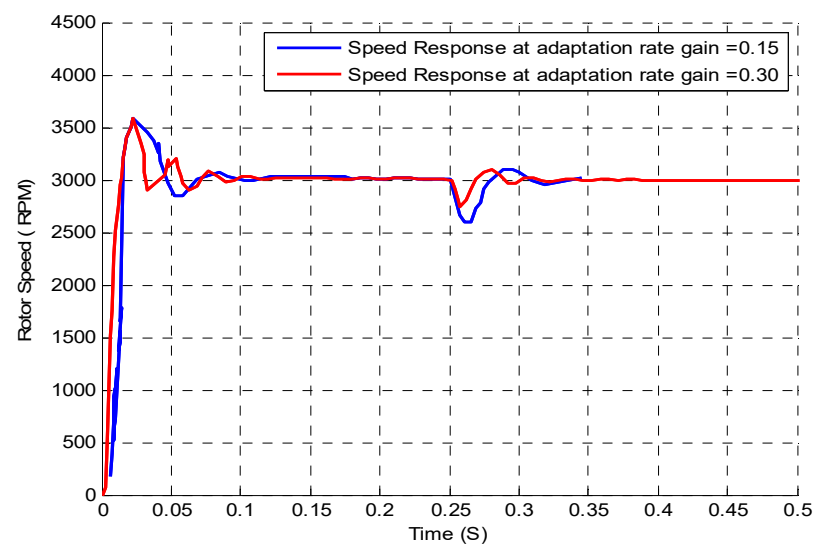

Figure 9. Speed response of the proposed controller for different adaptation rate gain

It can be inferred from the figure that proposed controller has better performance at sudden speed change when compared to the conventional controller. The rise time is less in the case of sudden change in load and hence the controller can act suitably fast to regulate the speed. This is of paramount importance as the proposed controller has the ability to recover the lost speed at a much faster rate. The controller output for the proposed controller and the conventional controller is illustrated in the Figure 10.

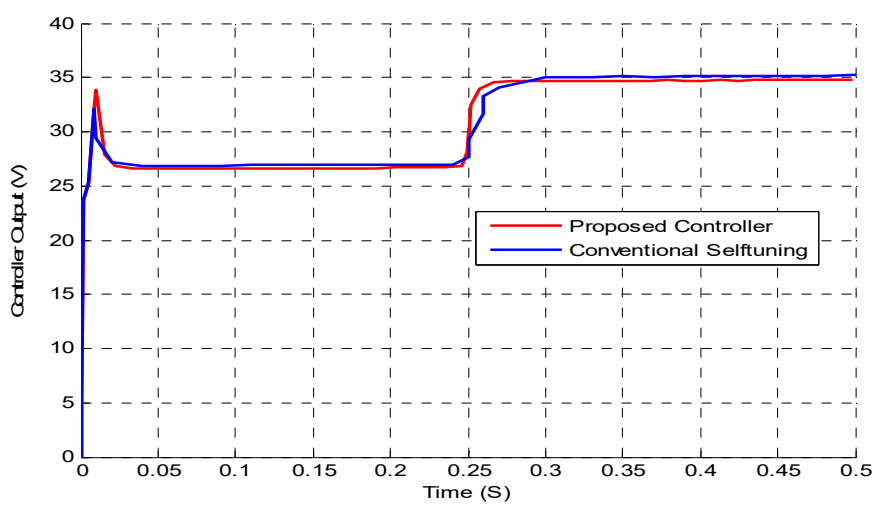

Figure 10. Comparison of controller output between the proposed and conventional self tuning PID control

In order to further evaluate the response of the proposed controller configuration it has been evaluated changing motor parameters like inertia and phase resistance individually and simultaneously. The inertia is increased by $15 \%$ while the phase resistance is decreased by $15 \%$. When simultaneous testing is made, both are increased by $50 \%$.

Figure 11 and Figure 12 illustrates and compares the performance of the proposed controller and conventional self tuning PID control when there is a sudden change in inertia. While Figure 11 depicts the speed response, Figure 12 presents the controller output. It can be observed from the figure that the proposed controller delivers a better response in comparison. 


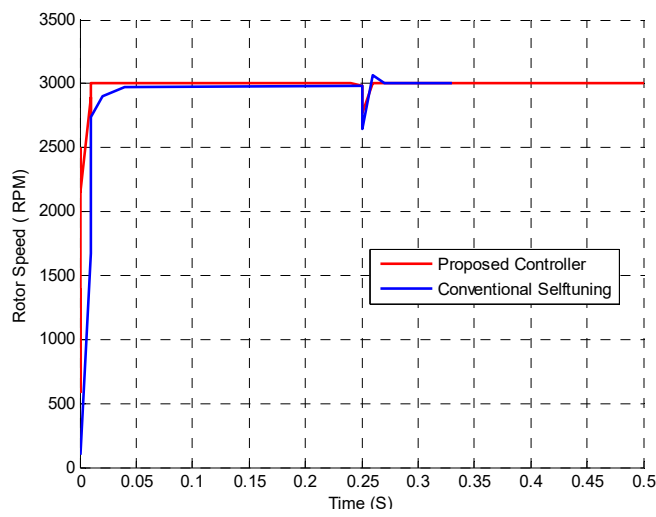

Figure 11. Speed control response with sudden change in inertia

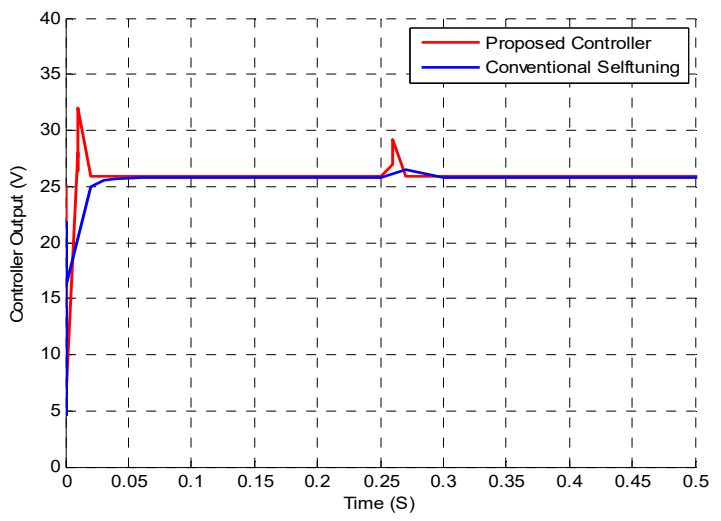

Figure 12. Controller output with sudden change in rotor inertia

Figure 13 depicts the controller output for sudden change in phase resistance; it can be observed from the figure that controller output varies rapidly with the change in inertia. The rise time is sudden and can help in enhancing the speed of response of the controller in maintain the constant speed.

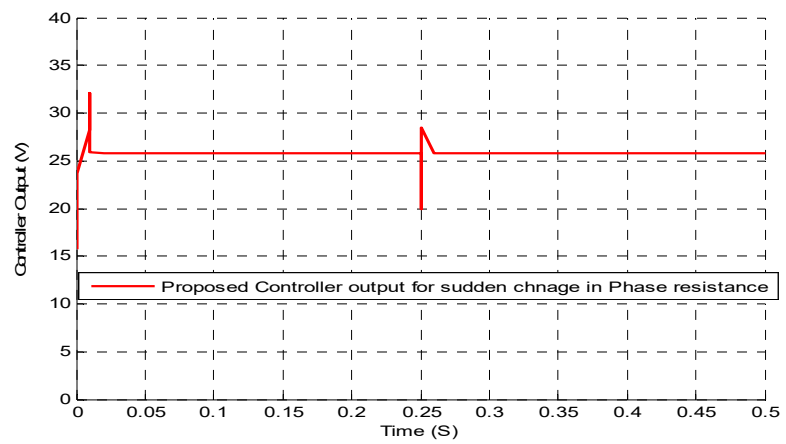

Figure 13. Controller output of the proposed controller for sudden change in phase resistance

The speed response of the proposed controller as compared with conventional self tuning controller is depicted using Figure 14. The comparison is made for 50\% change in phase resistance and inertia from the initial conditions. It can be observed from the figure there is no overshoot in the case of the proposed controller, where as in the case of conventional PID there is a significant overshoot from the reference value.

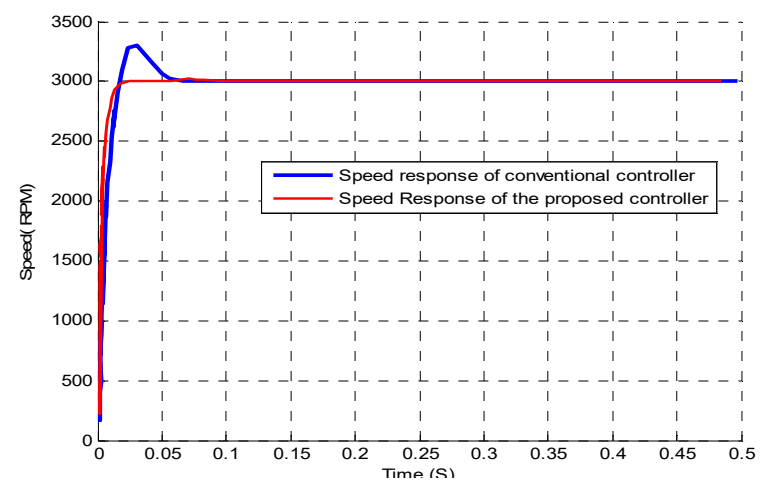

Figure 14. Speed control response for $50 \%$ change in phase resistance and $50 \%$ change in inertia 
In this case the motor speed is oscillated around the reference speed and the maximum speed deviation is observed. It can be observed from the Figure 15 that speed response is regulated efficiently with very little deviation in the maximum speed. This demonstrates the ability of the controller is suitably accommodating the load speed variations at the reference speed.

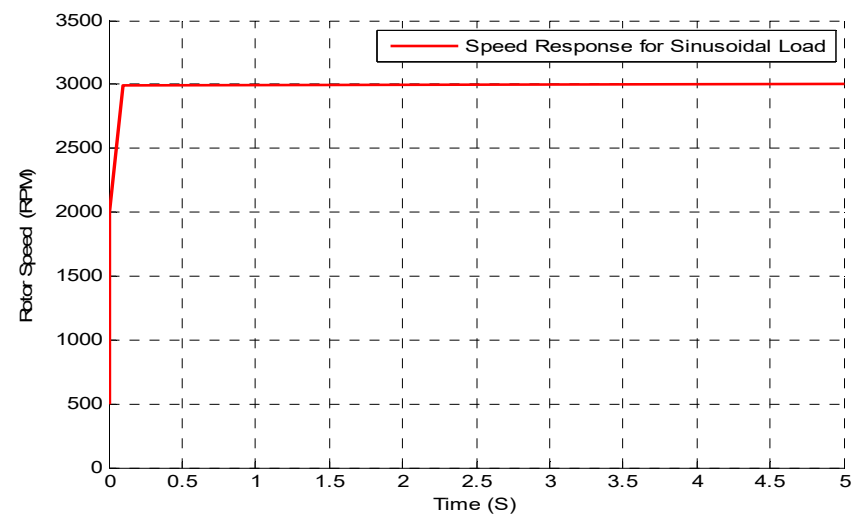

Figure 15. Speed control response of the proposed change controller for sinusoidal load

The Figure 16 illustrates the speed tracking response of the proposed controller. It shows the ability of the controller to track the changes in the set point. The reference speed is varied as step response and it can be observed from the figure that the controller is able to track the speed change without much of variation. This is observable across different speeds.

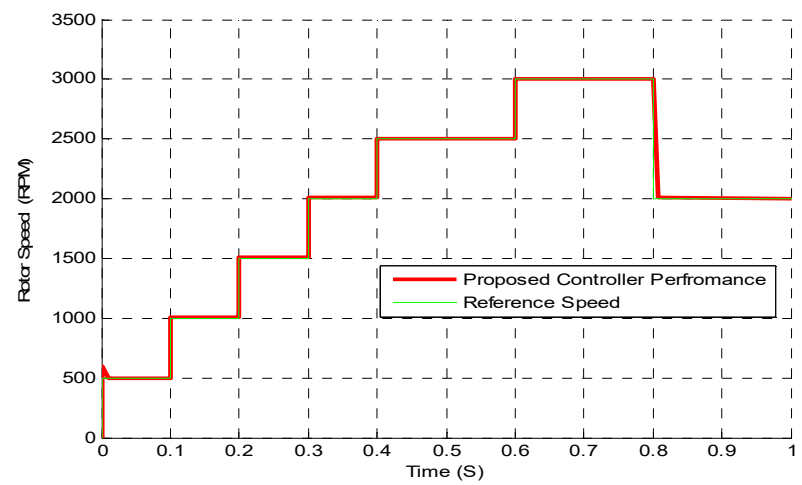

Figure 16. Speed tracking response of the proposed controller

\section{CONCLUSION}

A Model Reference Adaptive Control compensated with a GA- ANFIS tuned PID controller to control the modeled BLDC has been designed and presented. The results of tuning of the PID controller using GA-ANFIS depict an improved performance in terms of reduced overshoots, sudden change in load and settling time. Similarly the speed response of the controller for different adaptation rate gain has also been studied successfully compared with ANFIS .The proposed controller has also delivered better performance for sudden speed change, inertia change phase resistance change and proved its mettle in tracking sinusoidal change in the reference speed when compared to the conventional controller. In total the proposed controller has delivered a performance that is superior to the conventional self tuned PID controller. 


\section{REFERENCES}

[1] Kennedy, J., Eberhart, R., "Particle swarm optimization," In: Proceedings of IEEE International Conference on Neural Networks, pp. 1942-1948, 1995.

[2] Dorigo, M., Gambardella, L.M., "Ant Colony System: A Cooperative Learning Approach to the Traveling Salesman Problem". IEEE Transactions on Evolutionary Computation 1(1), 53-66, 1999.

[3] Holland, J.H., "Adaptation in Natural and Artificial Systems," University of Michigan Press, Ann Harbor, 1975.

[4] Ilhan Aydin, Mehmet Karakose, Erhan Akin, "A multi-objective artificial immune algorithm for parameter optimization in support vector machine," Applied Soft Computing, Volume 11, Issue 1, January 2011.

[5] A. Chatterjee and K. Watanabe, "An optimized Takagi- Sugeno type neuro-fuzzy system for modeling robot manipulators," Neural Computing \& Applications, vol. 15(1), pp. 55-61, 2006.

[6] M. A. Shoorehdeli, M. Teshnehlab and A. Sedigh, "Identification using ANFIS with intelligent hybrid stable learning algorithm approaches," Neural computing \& applications, vol. 18(2), pp. 157-174, 2009.

[7] M. A. Shoorehdeli, M. Teshnehlab and A. Sedigh, "Novel hybrid learning algorithms for tuning ANFIS parameters using adaptive weighted PSO," In Fuzzy Systems Conference, fuzzy-IEEE 2007, IEEE International , pp. 1-6, 2007.

[8] M. A. Shoorehdeli, M. Teshnehlab, A. K. Sedigh and M. A. Khanesar, "Identification using ANFIS with intelligent hybrid stable learning algorithm approaches and stability analysis of training methods," Applied Soft Computing, vol. 9(2), pp. 833-850, 2009.

[9] A. Z. Zangeneh, M. Mansouri, M. Teshnehlab and A. K. Sedigh, "Training ANFIS system with DE algorithm," In Advanced Computational Intelligence (IWACI), IEEE 2011 Fourth International Workshop on, pp. 308-314, October 2011.

[10] M. A. A. Ghany, M. A. Shamseldin, and A. M. A. Ghany, "A Novel Fuzzy Self Tuning Technique of Single Neuron PID Controller for Brushless DC Motor," International Journal of Power Electronics and Drive System (IJPEDS), vol. 8, no. 4, pp. 1705-1713, 2017.

[11] M Prita A., et al. "Comparative Study of Fuzzy Logic Based Speed Control of Multilevel Inverter fed Brushless DC Motor Drive". International Journal of Power Electronics and Drive System (IJPEDS), 4(1): 70-80, 2014.

[12] Lei Jin-li, "Adaptive Control for Brushless DC Motor Based on Fuzzy Inference". TELKOMNIKA Indonesian Journal of Electrical Engineering, 12(5): 3392-3398, 2014.

[13] M. A. Shamseldin, and A. M. A. Ghany, M. A. A. Ghany, "Performance Study of Enhanced Non-Linear PID Control Applied on Brushless DC Motor," International Journal of Power Electronics and Drive System (IJPEDS), vol. 9, no.2, pp. 536-545, 2018

[14] Murali Dasari,A Sreenivasula Reddy and M Vijayakumar, "Modeling of a commercial BLDC motor and control using GA-ANFIS tuned PID controller" - IEEE International Conference on Innovative Research In Electrical Sciences (IICIRES), 2017. 